\title{
TWO-DIMENSIONAL SLOSHING ANALYSIS BY THE ARBITRARY LAGRANGIAN-EULERIAN FINITE ELEMENT METHOD
}

\author{
Takashi OKAMOTO** \\ and Mutsuto KAWAHARA**
}

\begin{abstract}
This paper presents a new Arbitrary Lagrangian-Eulerian (ALE) finite element method for the calculation of a large amplitude sloshing wave in a tank with roofs and/or chamfers. For the analytical procedure, a new fractional step method to satisfy the free surface boundary condition has been applied, and the treatment of a free surface flow on the multi-sloped wall boundary has been advanced. Compared to experimental results, this method has proved to be sufficiently accurate to follow the free surface position and to obtain the velocity and pressure distributions. Furthermore, numerical examples show the validity to calculate large amplitude sloshing waves whose configuration of the free surface is double-valued function.

Keywords : tank, nonlinear sloshing analysis, free surface flow, Arbitrary LagrangianEulerian (ALE) method, finite element method
\end{abstract}

\section{INTRODUCTION}

Sloshing is a free surface flow problem in a tank which is subjected to forced oscillation. Clarification of the sloshing phenomena is very important in the design of the tank. A plenty of studies have been presented about the large amplitude sloshing analysis. Numerical methods presented previously for the sloshing analysis can be roughly classified into three methods, i.e., the finite difference method $^{1) \sim 7)}$, the boundary element method ${ }^{8) \sim 11)}$ and the finite element method ${ }^{12) 211}$. But the shapes of the tanks analyzed in almost all previous research has been limited to simple ones, i.e., rectangular or circular, and without roofs and/or chamfers. In the conventional numerical approach, there are two common ways of describing of the fluid motion. The first is the Eulerian description ${ }^{1)-6)}$, i.e., a coordinate is fixed in space and fluid moves through it. The second is the Lagrangian description ${ }^{12)-14)}$ in which a coordinate moves with the fluid. In addition to these descriptions, the Arbitrary Lagrangian-Eulerian method, i.e., reference frame moves with arbitrary velocity, has been proposed $^{7}$ and has been applied to the analysis of the fluid flow ${ }^{15)}$-21). In this paper, a new Arbitrary Lagrangian-Eulerian finite element method for the analysis of a free surface flow has developed, and its application to the large amplitude sloshing analysis in a tank which has complex configuration especially with roofs and chamfers is presented.

The main difficulties of computation in the large

* Member of JSCE, M. Eng., Senior Research Engineer, Steel Research Center, NKK Corporation (1-1, Minamiwatarida-cho, Kawasaki-ku, Kawasaki)

** Member of JSCE, Dr. Eng., Professor, Depertment of Civil Engineering, Chuo University amplitude sloshing analysis arise from the fact that both the calculations of an unknown free surface position and free surface condition should be satisfied simultaneously and the fact that the configuration of the fluid is strongly distorted. In the Lagrangian finite element method $^{12 \sim 14)}$, a coordinate moves with the fluid, which always agrees with the region to be analyzed. This is a great advantage in sloving the free surface problem. But, there is a disadvantage, which is related to the strong distrotion of the finite element mesh as a large amplitude sloshing. To overcome this point, the Arbitrary Lagrangian-Eulerian method is applied in this paper. In the analytical procedure of the present method, there are three phase procedures, i.e., Phase 1 : Purely Lagrangian calculation, Phase 2 : Remeshing procedure and Phase 3 : Rezoning procedure. In the Lagrangian calculation, a new fractional step method to satisfy the free surface boundary condition has been applied, and treatment of the free surface on the multi-sloped wall boundary has been advanced. In the remeshing procedure, a simple remeshing method which is adaptable to the multi-sloped wall boundary is introduced to avoid the strong irregular distortion of the mesh. Applying these three phase procedures, the present method overcomes the main difficulty of computation in the large amplitude sloshing analysis.

In order to investigate the accuracy of the present method, a comparison between the experimental and calculated results is performed, and a calculation of a large amplitude sloshing in a tank which has roofs and chamfers is carried out to verify the viability of the present method. The results show that the present method is sufficiently accurate and that the large amplitude slosing waves 
can be analyzed even if the configuration of a free surface is a double-valued function.

\section{ARBITRARY LAGRANGIAN-EULE. RIAN WINTTE ELEMENT METHOD}

\section{(1) ARBITRARY LAGRANGIAN - EULE- RIAN METHOD}

Any function $f$ of a physical property on a continuum medium has three types of time derivative, i.e., the material time derivative, the spatial time derivative and the referential time derivative, which are defined as follows :

$$
\begin{aligned}
& f_{t[X]}=\left.\frac{d f}{d t}\right|_{X_{t}=\text { const }} \\
& f_{t[x]}=\left.\frac{d f}{d t}\right|_{x_{t}=\text { const }} \\
& f_{t,[x]}=\left.\frac{d f}{d t}\right|_{x_{t}=\text { const }}
\end{aligned}
$$

where a comma followed by the subscripts denotes a partial derivative with respect to the following function and $X_{2}(i=1,2)$ are the material (Lagrangian) coordinates attached to the material, $x_{i}(i=1,2)$ are the spatial (Eulerian) coordinates fixed in space and $\chi_{2}(i=1,2)$ are the referential coordinates which are defined independent of material and spatial coordinates. The subscripted $X_{i}, x_{i}$, and $\chi_{i}$ accompanying the vertical bar in equations (2.1) $\sim(2.3)$ indicates the differentiation of $f$ that $X_{\imath}, x_{\imath}$ and $\chi_{\imath}$ are held constant.

The velocity of a material particle, $U_{t}$ is given by (2.4) and the referential velocity (or mesh velocity) $W_{t}$ is introduced to express the motion of a finite element mesh as in equation (2.5).

$$
\begin{aligned}
& U_{i}=x_{i, t[X]} \\
& W_{\imath}=x_{i, t[x]}
\end{aligned}
$$

The convective velocity $C_{l}$, which is the difference between the material and mesh velocities is expressed as follows,

$$
C_{i}=U_{i}-W_{i}
$$

The relationship between the time derivative with respect to material and referential functions is formulated in the following form.

$$
f_{, t[X]}=f_{, t[x]}+f_{, t} C_{t}
$$

Equation (2.7) is an important relationship in the Arbitrary Lagrangian-Eulerian method, the meanings of which are :

(1) If $W_{t}=U_{t}, C_{t}=0$, the reference frame moves in space with the same velocity as the particles. Equation (2.7) is reduced to $f_{t,[X]}=f_{t[x]}$. This corresponds to the Lagrangian description.

(2) If $W_{t}=0, C_{t}=U_{t}$, the reference frame is fixed in space. This corresponds to the Eulerian description.

(3) If $W_{\imath} \neq U_{\imath} \neq 0, C_{\imath} \neq U_{i} \neq 0$, the reference

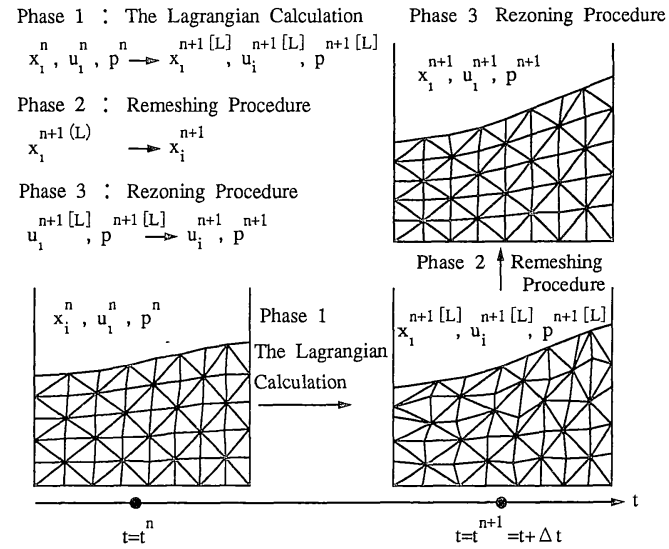

Fig.2.1 Three phases in the Arbitrary LagrangianEulerian finite element method

frame moves in space with an arbitrary velocity. This is the Arbitrary Lagrangian-Eulerian method.

Three phases will be employed in the Arbitrary Lagrangian-Eulerian method which are schematically shown in Fig.2.1. The total time to be analyzed is divided into short time increments one of which is denoted by $\Delta t$. At the $n$-th time point, the analysis is carried out using the following procedures.

Phase 1: The Lagrangian calculation : Calculate the $(n+1)$-th value using the $n$-th time result based on the Lagrangian method.

$$
x_{i}^{n}, U_{t}^{n}, p^{n} \rightarrow x_{i}^{n+1[L]}, U_{t}^{n+1[L]}, p^{n+1[L]}
$$

Phase 2 : Remeshing procedure : To avoid the strong irregular distortion of the mesh in the Lagrangian calculation, re-generate a new finite element mesh. The referential velocity (mesh velocity) is specified in this phase.

$$
x_{\imath}{ }^{n+1[L]} \rightarrow x_{\imath}{ }^{n+1}
$$

Phase 3 : Rezoning procedure : Calculate the $(n$ $+1)$-th value to be compatible with a new mesh.

$$
U_{i}^{n+1[L]}, p^{n+1[L]} \rightarrow U_{i}^{n+1}, p^{n+1}
$$

\section{(2) LAGRANGIAN CALCULATION}

\section{(PHASE 1)}

\section{a) BASIC EQUATION}

The equations of motion and continuity in the Lagrangian description are expressed as follows,

$$
\begin{aligned}
& D U_{\imath} / D t=-p_{, 2} / \rho+\nu\left(U_{\imath, j}+U_{,, 2}\right)_{,}+f_{t} \cdots \\
& U_{i, 2}=0
\end{aligned}
$$

where $U_{i}$ and $f_{i}$ are velocity and acceleration in the $x_{i}$-direction $(i=1,2), p$ is pressure, $\rho$ and $\nu$ are density and kinematic viscosity respectively. $D U_{i} / D t$ means the material derivative of velocity. The following two types of boundary conditions are used in this analysis. One is assumed to be the boundary on the rigid wall (on $S_{1}$ ) and the other is 
the boundary on the free surface (on $S_{2}$ ).

$$
U_{i}=\widehat{U}_{i} \text { on } S_{1} \text { (on the rigid wall) }
$$

$$
\left\{-p \delta_{i j}+\nu\left(U_{t, j}+U_{\jmath, t}\right)\right\}_{n}=\widehat{T}_{i}=0
$$

$$
\text { on } S_{2} \text { (on the free surface) }
$$

where $\widehat{U}_{\imath}$ and $\widehat{T}_{\imath}$ are given values on the boundaries, and $\delta_{i j}$ is the Kronecker delta, $n$, is the $j$-th component of the outward unit normal vector respectively.

\section{b) FRACTIONAL STEP METHOD}

In the Lagrangian calculation, the material acceleration is approximated as follows :

$$
D U_{t} / D t \cong\left(U_{\imath}^{n+1[L]}-U_{t}^{n}\right) / \Delta t
$$

where

$$
\begin{aligned}
& U_{\imath}{ }^{n}=U_{t}\left(t_{n}, x_{\imath}{ }^{n}\right) \ldots \ldots \ldots \ldots \ldots \ldots \\
& U_{\imath}{ }^{n+1[L]}=U_{\imath}\left(t_{n}+\Delta t, x_{\imath}{ }^{n+1[L]}\right) \\
& x_{\imath}{ }^{n+1[L]}=x_{\imath}{ }^{n}+\Delta t U_{t}{ }^{n+1[L]} \ldots \ldots . .
\end{aligned}
$$

in which $x_{t}^{n}$ and $x_{i}{ }^{n+1[L]}$ are positions of the fluid particle at time points $t^{n}$ and $t^{n+1}=t^{n}+\Delta t$, and $U_{i}^{n}$ and $U_{l}^{n+1[L]}$ are the particle velocities at time $t^{n}$ and $t^{n+1}=t^{n}+\Delta t$ in the Lagrangian calculation, and superscript $[L]$ means that the function is obtained by the Lagrangian calculation. The new fractional step method is originated based on the Lagrangian formulation. The calculation procedures are as follows :

(1) Pressure is obtained by the implicit scheme i.e., by the solution of the Poisson equation for $p^{n+1}$ as follows.

$$
\begin{aligned}
p_{. \imath 2}{ }^{n+1[L]}= & (1 / \Delta t) U_{t, 2}{ }^{n}+\nu\left(U_{t, 3}{ }^{n+1[L]}\right. \\
& \left.+U_{,, 2}{ }^{n+1[L]}\right)_{,, 2}+f_{t, \imath}{ }^{n+1} \ldots \ldots
\end{aligned}
$$

(2) Velocity is computed by the explicit scheme :

$$
\begin{aligned}
U_{t}{ }^{n+1[L]}= & U_{\imath}{ }^{n}-\Delta t\left\{p_{, \imath}{ }^{n+1[L]}-\nu\left(U_{t, j}{ }^{n+1[L]}\right.\right. \\
& \left.\left.+U_{,, 2}{ }^{n+1[L]}\right),{ }_{, j}+f_{\imath}{ }^{n+1}\right\} \ldots \ldots \ldots \ldots . .
\end{aligned}
$$

Because in the right-hand side of equations (2.16) and (2.17) unknown value $U_{t}^{n+1[L]}$ is included, these equations should be solved by an iterative procedure.

\section{c) FINITE ELEMENT FORMULATION}

Multiplying both sides of equations (2.16) and (2.17) by the weighting functions $U_{2}^{*}$ and $p^{*}$ respectively and integrating over the domain $V_{L}$, applying the integration by parts and using the divergence theorem, the weighted residual equations are derived in the following forms.

$$
\begin{aligned}
& \int_{V}\left(p_{, 2}{ }^{*} p_{, \imath}{ }^{n+1[L]}\right) d V_{L} \\
& =-(1 / \Delta t) \int_{V}\left(p^{*} U_{l, 2}{ }^{n}\right) d V_{L}
\end{aligned}
$$

$$
\begin{aligned}
& +\nu \int_{{ }_{,}}\left(U_{t, j}{ }^{n+1[L]}+U_{,, l}{ }^{n+1[L]}\right),{ }_{,} d V_{L} \\
& +\int_{V}\left(p^{*}{ }_{,} f_{l}^{n+1}\right) d V_{L} \\
& +\int_{S} p^{*}\left\{\left(U_{\imath}{ }^{n+1}-U_{\imath}{ }^{n}\right) / \Delta t\right\} n_{t} d S_{L}
\end{aligned}
$$

$$
\begin{aligned}
& \int_{V}\left(U_{i}^{*} U_{i}^{n+1[L]}\right) d V_{L} \\
& =\int_{V}\left(U_{\imath}^{*} U_{\imath}^{n}\right) d V_{L}+\Delta t\left[\int_{V}\left(U_{\imath, 2} p^{n+1[L]}\right) d V_{L}\right. \\
& -\nu \int_{V} U_{i, j} *\left(U_{i, j}{ }^{n+1[L]}+U_{j, 2}{ }^{n+1[L]}\right) d V_{L} \\
& -\int_{V}\left(U_{\imath}^{*} f_{\imath}^{n}\right) d V_{L}+\int_{S} U_{\imath}^{*}\left\{-p^{n+1[L]} \delta_{\imath\}}\right. \\
& \left.\left.+\nu\left(U_{\imath, \rho}{ }^{n+1[L]}+U_{j,{ }^{n+1}}{ }^{n[L]}\right)\right\} n_{\jmath} d S_{L}\right]
\end{aligned}
$$

The boundary conditions for equations (2.18) and (2.19) are as follows:

$$
\begin{aligned}
& \left\{\left(U_{\imath}^{n+1[L]}-U_{\imath}^{n}\right) / \Delta t\right\} n_{\imath}=\widehat{Q} \\
& \text { on } S_{1} \text { (on the rigid wall) } \\
& p^{n+1[L]}=p \\
& \text { on } S_{2} \text { (on the free surface) } \cdots \cdots(2.21) \\
& U_{i}^{n+1[L]}=\widehat{U}_{i} \\
& \text { on } S_{1} \text { (on the rigid wall) } \\
& \left\{-p^{n+1[L]} \delta_{i j}+\nu\left(U_{i, j}{ }^{n+1[L]}+U_{j, 2}{ }^{n+1[L]}\right)\right\} n_{j}=\widehat{T}_{\imath}=0 \\
& \text { on } S_{2} \text { (on the free surface) } \cdots \cdots(2.23)
\end{aligned}
$$

Equation (2.20) and (2.22) are the boundary conditions on the rigid wall, and equation (2.21) and (2.23) are the boundary conditions on the free surface.

The pressure will be given by equation (2.21) as the boundary condition on the free surface in equation (2.18). Boundary condition (2.21) does not correspond to the boundary condition on the free surface which is given by equation (2.11). Therefore, the pressure $p^{n+1[L]}$ on a free surface is calculated to satisfy the equation (2.11) from the velocity $U_{t}^{n+1[L]}$, and the pressure is given on a free surface to satisfy the boundary condition. The pressure is calculated by the explicit method as shown in equation (2.24).

$$
\begin{aligned}
p^{n+1[L]}= & \left\{2 \nu /\left(n_{1}{ }^{2}+n_{2}{ }^{2}\right)\right\}\left\{U_{1,1} n_{1}{ }^{2}+U_{2,2} n_{2}{ }^{2}\right. \\
& \left.+\left(U_{1,2} U_{2,1}\right) n_{1} n_{2}\right\} \ldots \ldots \ldots \ldots \ldots \ldots \ldots
\end{aligned}
$$

where $n_{1}$ and $n_{2}$ are components of a outward unit normal in the direction of $x_{1}$ and $x_{2}$, respectively.

Velocity $U_{t}$, pressure $p$ and the corresponding weighting functions $U_{1}^{*}, p^{*}$ are interpolated in each finite element as follows :

$$
U_{\imath}=\Phi_{\alpha} U_{t \alpha}, \quad U_{t}{ }^{*}=\Phi_{\alpha} U_{t \alpha}{ }^{*}
$$




$$
p=\Phi_{\alpha} p_{\alpha}, \quad p^{*}=\Phi_{\alpha} p_{\alpha}^{*}
$$

where $\bar{\Phi}_{\alpha}$ denotes the interpolation function for both velocity and pressure. Quantities $U_{\alpha l}$ and $p_{\alpha}$ are nodal values of velocity and pressure at $\alpha$ th node of the finite element respectively, and $U_{\alpha i}{ }^{*}$ and $p_{\alpha}{ }^{*}$ are the nodal values of the weighting functions $U_{i}^{*}$ and $p^{*}$ respectively. The standard linear interpolation function based on the three node triangular finite element are used.

Substituting equations (2.25) and (2.26) into equations (2.18) and (2.19), the finite element equations (2.27) and (2.28) can be derived as follows.

$$
\begin{aligned}
& A_{\alpha \imath \beta} p_{\beta}{ }^{n+1[L](m+1)} \\
& =-(1 / \Delta t) H_{\alpha \beta \imath} U_{\beta \imath}{ }^{n}+F_{\alpha \imath \beta} f_{\beta \imath}{ }^{n+1}+\widehat{\Sigma}_{\alpha} \\
& \bar{M}_{\alpha \beta} U_{\beta 2}{ }^{n+1[L](m+1)} \\
& =\bar{M}_{\alpha \beta} U_{\beta \imath}{ }^{n}+\Delta t\left\{F_{\alpha \imath \beta} p_{\beta}{ }^{n+1[L](m+1)}\right. \\
& \left.-S_{\alpha \imath \beta,} U_{\beta}{ }^{n+1[L](m)}+N_{\alpha \beta} f_{\beta t}{ }^{n+1}+\widehat{\Omega}_{\alpha \imath}\right\}
\end{aligned}
$$

where

$$
\begin{aligned}
& A_{\alpha \imath \beta}=\int_{V}\left(\Phi_{\alpha, \imath} \bar{\Phi}_{\beta, \imath}\right) d V_{L} \\
& H_{\alpha \beta}=\int_{V}\left(\Phi_{\alpha} \bar{\Phi}_{\beta, 2}\right) d V_{L} \\
& F_{\alpha \imath \beta}=\int_{V}\left(\Phi_{\alpha, 2} \bar{\Phi}_{\beta}\right) d V_{L} \\
& M_{\alpha \beta}=\int_{V}\left(\Phi_{\alpha} \bar{\Phi}_{\beta}\right) d V_{L} \\
& S_{\alpha \imath \beta \jmath}=\nu\left\{\int_{V}\left(\Phi_{\alpha, k} \Phi_{\beta, k} \delta_{\imath \jmath}\right) d V_{L}+\int_{V}\left(\bar{\Phi}_{\alpha, l} \bar{\Phi}_{\beta, \jmath}\right) d V_{L}\right\} \\
& N_{\alpha \beta}=\int_{V}\left(\Phi_{\alpha} \Phi_{\beta}\right) d V_{L} \\
& \widehat{\Sigma}_{\alpha}=\int_{S} \Phi_{\alpha}\left\{\left(U_{t}^{n+1[L]}-U_{\imath}^{n}\right) / \Delta t\right\} n_{t} d S_{L} \\
& =\int_{S}\left(\Phi_{\alpha} \widehat{Q}\right) d S_{L} \\
& \widehat{\Omega}_{\alpha 1}=\int_{S} \Phi_{\alpha}\left\{-p^{n+1[L]} \delta_{\imath \jmath}+\nu\left(U_{\imath, j}{ }^{n+1[L]}\right.\right. \\
& \left.+U_{\left.j, l^{n+1[L]}\right)}\right\}_{n,} d S_{L}=\int_{S}\left(\bar{\Phi}_{\alpha} \widehat{T}_{i}\right) d S_{L}=0
\end{aligned}
$$

$\bar{M}_{\alpha \beta}$ in equation (2.28) is the lumped coefficient obtained from $M_{\alpha \beta}$.

d) CALCULATION PROCEDURE

The calculation procedures for this method at $t=$ $t^{n}$ time point are summarized as follows :

(1) Assign $U_{2}{ }^{n+1[L](m)}=U_{i}^{n}$.

(2) Calculate new position of the nodal point by $x_{t}^{n+1(m)}=x_{t}^{n}+\Delta t U_{t}^{n+1(m)}$.

(3) Solve the Poisson equation (2.27) for

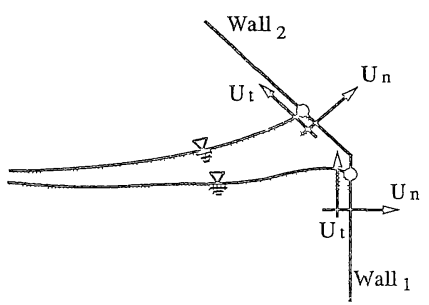

Fig.2.2 Treatment of sloped or multi-sloped wall boundary

$$
p^{n+1(m+1)} \text { using } U_{i}^{n+1(m)} \text { and } U_{l}^{n} \text {. }
$$

(4) Calculate $U_{i}^{n+1(m+1)}$ by equation (2.28) using $p^{n+1(m+1)}, U_{i}^{n}$ and $U_{i}^{n+1(m)}$.

(5) Examine whether $U_{2}^{n+1(m+1)}$ converges sufficiently close to $U_{t}^{n+1(m)}$ or not and if not replace $U_{l}^{n+1(m)}$ by $U_{l}^{n+1(m+1)}$ and recalculate from step (2).

(6) Continue to calculate until the preassigned final time.

In this procedure, $(m)$ means $m$-th iteration.

e) SLOSHING WAVE ON SLOPED WALL

In the calculation of a large amplitude sloshing wave in a tank which has roof or chamfer, it is necessary to treat the sloped or multi-sloped wall boundary. The treatment of this boundary is shown in $\mathbb{F}_{\mathrm{ig}}$.2.2. The procedure is summarized as follows.

(1) Judge on which sloped wall the boundary nodal point lies.

(2) Calculate the components of a unit normal to the boundary wall surface.

(3) Compute the tangential velocity $U_{t}$ of the boundary nodal point and use $U_{t}$ to determine the new position of the nodal point.

(4) Set the component of velocity normal to the wall $U_{n}$ to be zero.

For the analysis of a viscous fluid in general, the boundary condition at the wall is sometimes nonslip boundary. i.e., both normal and tangential components of the velocity are set to be zero. However, in this sloshing analysis, due to the finite element size, which is not enough to resolve the boundary layer, a free slip approximation is used on the wall of the tank. In this paper, an explicit type of numerical integration in time is used. In the scheme. $U_{t}$ and $U_{n}$ are corrected at each time step.

As shown in $\mathbb{F i g . 2 . 3 , ~ w h e n ~ n o d a l ~ p o i n t s ~ c r o s s ~ a ~}$ corner of multi-sloped wall boundary, an artificial cave occures between wall and finite element. In the present calculation, it is assumed that the cave is so small that it can be negligibly small.

f) FORCED OSCILLATION OF TANKS

There are two ways of imposing a forced 


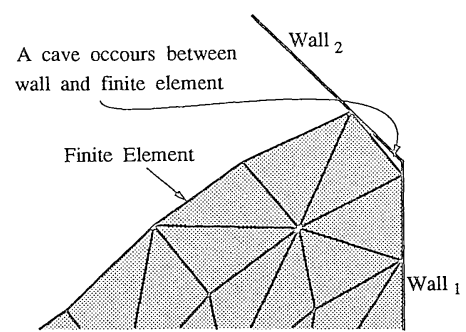

Fig.2.3 Nodal points cross a corner of multi-sloped wall boundary

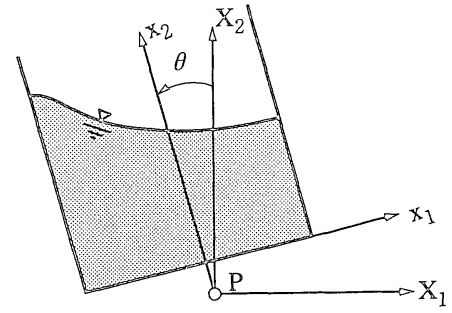

Fig.2.4 Forced oscillation of tanks

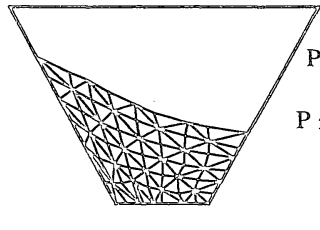

(a)

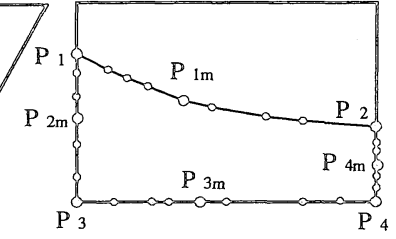

(b)

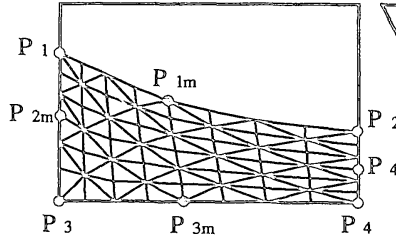

(c)

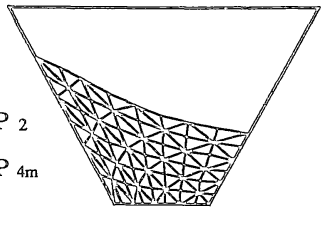

(d)

Fig.2.5 Remeshing procedure

oscillation of tanks. In the first one the acceleration effect of the tank is taken into account as the $f_{i}$ in equation (2.8) and in the second one the velocity of the wall is imposed as the boundary condition.

Coordinates axes $X_{1}-X_{2}$ are fixed in space, and coordinates axes $x_{1}-x_{2}$ are fixed to the tank, and $x_{1}$ is chosen to correspond to the tank bottom and $x_{2}$ is chosen to correspond to the center of the tank as shown in Fig.2.4. Let a tank be oscillated by the rotating motion around point $\mathbb{P}$, horizontal and perpendicular motions along $X_{1}$ and $X_{2}$-axes. In the first case, accelerations are assumed to be given by

$$
\begin{aligned}
f_{x_{1}}= & -g \sin \theta+2 \dot{\theta} U_{2}+\ddot{\theta}\left(x_{2}-R_{x_{2}}\right)+\dot{\theta}^{2}\left(x_{1}-R_{x_{1}}\right) \\
& -\ddot{X}_{1} \cos \theta-\ddot{X}_{2} \sin \theta \cdots \cdots \cdots \cdots \cdots \cdots \cdots(2.37) \\
f_{x_{2}}= & -g \cos \theta-2 \dot{\theta} U_{1}-\ddot{\theta}\left(x_{1}-R_{x_{1}}\right)+\dot{\theta}^{2}\left(x_{2}-R_{x_{2}}\right) \\
& +\ddot{X}_{1} \sin \theta-\ddot{X}_{2} \cos \theta \cdots \cdots \cdots \cdots \cdots \cdots \cdots(2.38)
\end{aligned}
$$

where, $g$ is gravity acceleration. $R_{x_{1}}$ and $R_{x_{2}}$ are coordinates of point $P$ which is the center of the rotation. Angular displacement $\theta$ is positive counterclockwise. Notation' denotes the differentiation with respect to time. In the second case, velocity on the wall is assumed to be given by

$$
U_{1}=-\left(x_{2}-R_{x_{2}}\right) \dot{\theta}+\dot{X} \cos \theta+\dot{Y} \sin \theta
$$

(on the side wall)

$$
U_{2}=\left(x_{1}-R_{x_{1}}\right) \dot{\theta}-\dot{X} \sin \theta+\dot{Y} \cos \theta
$$

(on the bottom)

where $X_{1}, X_{2}, x_{1}, x_{2}, R_{x_{1}}, R_{x_{2}}$ are the same as in equations (2.37) and (2.38).

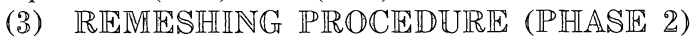

In the ALE method, the remeshing procedure plays an important role in continuing the stable computaion avoiding the strong irregular distortion of the mesh. In this paper, a simple remeshing method which can be applied to the multi-sloped boundary is used. The outline of the remeshing procedure is shown in $\mathbb{F} i g .2 .5$. Fig.2.5 (a) shows the finite element mesh before applying the remeshing procedure in the analysis of the tank with a sloped wall boundary. Mapping transformation is done in such a way that this mesh is mapped to the shape of a rectangular region. This is the transformation that both sloped walls move into the perpendicular ones. The outlines after the transformation are shown in $\mathbb{F} i \mathrm{~g} .2 .5$ (b). The mesh is generated based on these outlines. Consider a free surface. The number of mesh divisions is $N$, and node $P_{m 1}$ is a node for the control point of the remeshing procedure. The length on a free surface is divided into $N$ interpolating segments by a quadratic function with node $P_{1}, P_{1 m}$ and $P_{2}$ as shown in Fig.2.5 (c). At this time, it becomes the equal division when the middle point is not considered. When the free surface configuration is complex, the length on a free surface is divided using a third order polynomials with the two middle control points. Other sides are also operated in a similar way. Auxiliary grids are made by connecting the node of each side. The reverse mapping transformation is made, and the remeshing procedure is completed. Finally, the new coordinate value $x_{i}^{n+1}$ is obtained. Using the new coordinate value $x_{i}^{n+1}$, mesh velocity $W_{i}$ and convective velocity $C_{i}$ are calculated in the following form,

$$
W_{i}=\left(x_{i}{ }^{n+1}-x_{i}{ }^{n}\right) / \Delta t \cdots \cdots \cdots \cdots \cdots \cdots \cdots \cdots \cdots \cdots \cdots(2.41)
$$




$$
C_{i}=U_{i}^{(n+1)[L]}-W_{i}
$$

(4) REZONING PROCEDURE (PHASE 3)

The velocity and pressure at the $(n+1)$-th time point in the Lagrangian calculation are transformed to adapt the re-generation of a new mesh. The velocity and pressure should be transformed to the corresponding positions using the relation of the time derivative shown in equation (2.12), of which weighted residual forms are :

$$
\begin{gathered}
\int_{V}\left(U_{i}^{*} U_{i}^{n+1}\right) d V_{n+1}=\int_{V}\left(U_{i}^{*} U_{i}^{(n+1)[L]}\right) d V_{n+1} \\
-\int_{V} \Delta t\left(U_{i}^{*} U_{i, j}{ }^{(n+1)[L]} C_{\jmath}\right) d V_{n+1} \cdots(2.43) \\
\int_{V}\left(p^{*} p^{n+1}\right) d V_{n+1}=\int_{V}\left(p^{*} p^{(n+1)[L]}\right) d V_{n+1} \\
-\int_{V} \Delta t\left(p^{*} p_{, j}{ }^{(n+1)[L]} C_{\jmath}\right) d V_{n+1}
\end{gathered}
$$

The kinematic boundary condition on a free surface is always satisfied in the Lagrangian calculation. In cases when the ALE method is applied, the node on a free surface must be corrected to satisfy the kinematic boundary condition. This correction can be obtained by the following equation.

$$
\begin{aligned}
\int_{S}\left(x_{i}{ }^{*} x_{\imath}{ }^{n+1}\right) d S_{n+1}= & \int_{S}\left(x_{i}{ }^{*} x_{\imath}{ }^{(n+1)[L]}\right) d S_{n+1} \\
& -\int_{S} \Delta t\left(x_{i}{ }^{*} x_{\imath, j}{ }^{(n+1)[L]} C_{\jmath}\right) d S_{n+1}
\end{aligned}
$$

From the weighted residual equations of (2.43), (2.44) and (2.45), $U_{i}^{n+1}, p^{n+1}$ and $x_{i}{ }^{n+1}$ can be calculated. The finite element equations for these equations are obtained as follows.

$$
\begin{aligned}
& \bar{M}_{\alpha \beta} U_{\beta \imath}{ }^{n+1}=\bar{M}_{\alpha \beta} U_{\beta t}{ }^{n+1[L]}-\Delta t S_{\alpha \beta \jmath \gamma} U_{\beta i}{ }^{n+1[L]} C_{\gamma} \\
& \text { ….............................. (2.46) } \\
& \bar{M}_{\alpha \beta} p_{\beta}{ }^{n+1}=\bar{M}_{\alpha \beta} p_{\beta}{ }^{n+1[L]}-\Delta t S_{\alpha \beta \gamma \gamma} p_{\beta}{ }^{n+1[L]} C_{\gamma \jmath} \\
& \text {................................ (2.47) } \\
& \bar{N}_{\alpha \beta} x_{\beta \imath}{ }^{n+1}=\bar{N}_{\alpha \beta} x_{\beta \imath}{ }^{n+1[L]}-\Delta t H_{\alpha \beta \jmath \gamma} x_{\beta \imath}{ }^{n+1[L]} C_{\gamma}
\end{aligned}
$$

"where

$$
\begin{aligned}
M_{\alpha \beta} & =\int_{V}\left(\Phi_{\alpha} \Phi_{\beta}\right) d V_{n+1} \cdots \ldots . \\
S_{\alpha \beta \jmath \gamma} & =\int_{V}\left(\Phi_{\alpha} \Phi_{\beta, \jmath} \Phi_{\gamma}\right) d V_{n+1} \cdots \\
N_{\alpha \beta} & =\int_{S}\left(\Phi_{\alpha} \Phi_{\beta}\right) d S_{n+1} \ldots \ldots \ldots \\
H_{\alpha \beta \gamma \gamma} & =\int_{S}\left(\Phi_{\alpha} \Phi_{\beta, \jmath} \bar{\Phi}_{\gamma}\right) d S_{n+1}
\end{aligned}
$$$$
\bar{M}_{\alpha \beta} \text { and } \bar{N}_{\alpha \beta} \text { in equations (2.46), (2.47) and }
$$
(2.48) are the lumped coefficients obtained from $M_{\alpha \beta}$ and $N_{\alpha \beta}$ respectively.

\section{COMPARISON BETWEEN COM- PUTED AND EXPERIMENTAL RE- SULTS}

To investigate the validity of the present method, a comparison is made between the computed results and the experimental results.

(1) COMPARISON WITH EXPERTMENT OF A RECTANGULAR TANK (1)

A transparent acrylic resin tank was used in the experiment which was $1000 \mathrm{~mm}$ in width, 1200 $\mathrm{mm}$ in height and $100 \mathrm{~mm}$ in breadth as shown in Fig.3.1. Water was used and the depth was 500 mm. The tank was set on a shaking table which oscillated in a sinusoidal function of time starting from the zero velocity state. Sloshing phenomena were recorded using a video camera.

The finite element model is shown in Fig.3.1. The analytical region is divided into the mesh of 24 $\times 20$. The density is $1.0 \mathrm{Mg} / \mathrm{m}^{3}$ and the coefficient of kinematic viscosity is $\nu=1.0 \times 10^{-2} \mathrm{~cm}^{2} / \mathrm{sec}$. Acceleration $f_{2}$ is $g=-9.80 \mathrm{~m} / \mathrm{sec}^{2}$. Amplitude and period of displacement of the table are $A_{x_{1}}=$ $9.3 \mathrm{~mm}$ and $T_{x_{1}}=1.183 \mathrm{sec}\left(\omega_{x_{1}}=5.311 \mathrm{rad} / \mathrm{sec}\right)$, respectively. The period is nearly equal to the first characteristic period calculated by the linear potential theory. The time increment $\Delta t$ is $2.0 \times$ $10^{-3} \mathrm{sec}$. Boundary conditions are shown in Fig.3.1.

Fig.3.1 (a) and (b) show both the experimental and calculated results at time $t=3.54 \mathrm{sec}$ and $t=$ $7.08 \mathrm{sec}$ respectively. In these figures, the left column shows the experimental result, while the right three columns show the calculated results of the free surface, finite element configuration and pressure distribution. The calculated configurations of the free surface agree well with the experimental results. The ratio of the sloshing wave height to the water depth $\eta / h$ is 0.20 at time $t=$ $3.54 \mathrm{sec}$ and 0.55 at time $t=7.08 \mathrm{sec}$ respectively. In such cases where that $\eta / h$ is larger than 0.20 , the computation is impossible in the Lagrangian finite element method ${ }^{14)}$ because of the strong distortion of the mesh. If the ALE method is applied, the computation is which $\eta / h$ is larger than 0.20 is stable even if the time increment is 2 times as long as that in the Lagrangian finite element mehtod.

\section{(2) COMPARISON WITH EXPERIMENT OF \\ A RECTANGULAR TANK (2)}

A transparent acrylic resin tank was used in the experiment which was $1060 \mathrm{~mm}$ in width, $756 \mathrm{~mm}$ in height and $200 \mathrm{~mm}$ in breadth as shown in Fig.3.2. Water was used and the depth was 151 $\mathrm{mm}$. The tank was set on a oscillation simulator and the tank was oscillated by the rotating motion. 


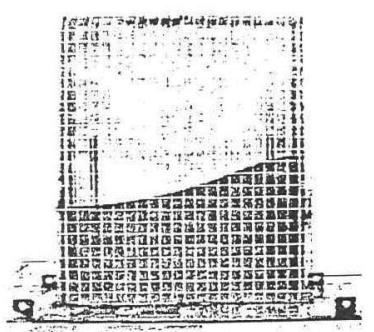

Experimental Result

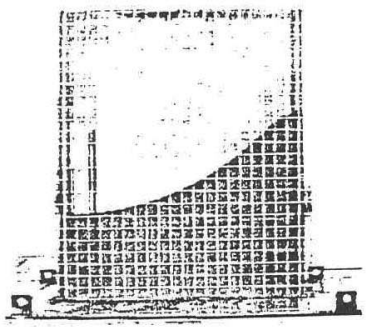

Experimental Result

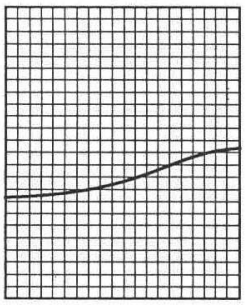

Free surface

(a) At time $t=3.54 \mathrm{sec}$

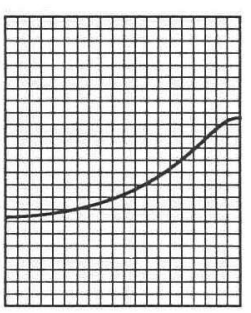

Free surface

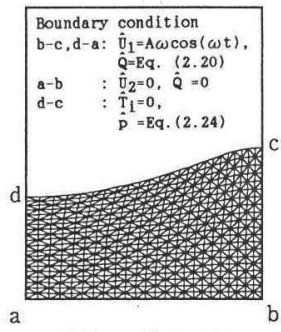

F.E. configuration Calculated result

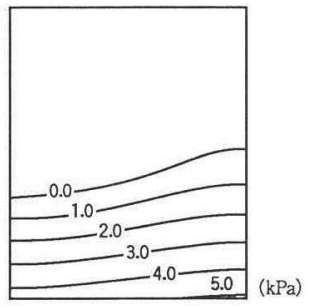

Pressure distribution

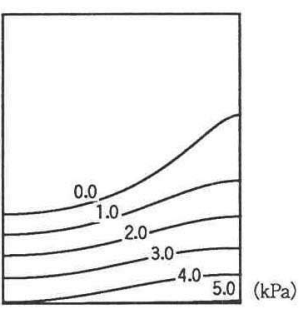

Pressure distribution

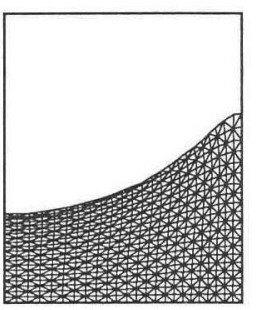

F.E. configuration Calculated result

(b) At time $t=7.08 \mathrm{sec}$

Fig.3.1 Experimental and calculated results
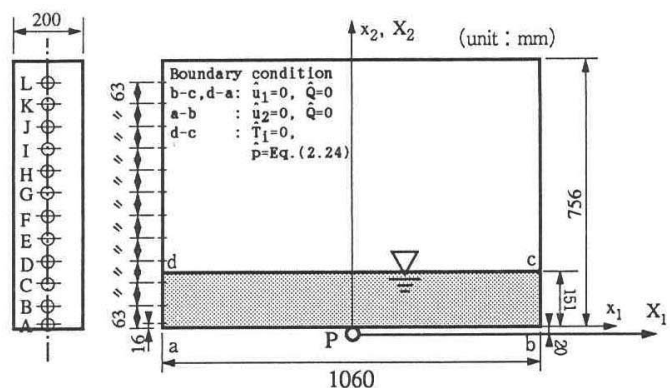

Fig.3.2 Model tank and arrangement of the pressure sensors

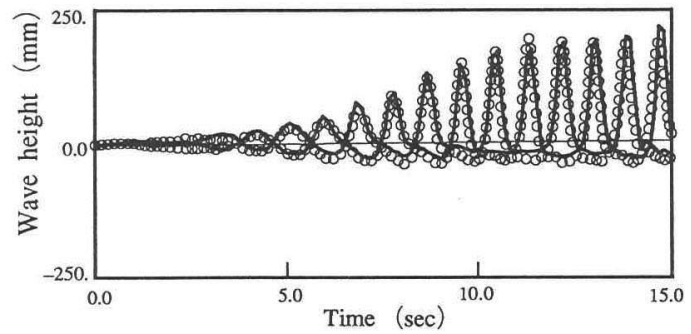

Fig.3.3 Wave height of the right and left walls

The tank rotated around point $\mathbf{P}$. The condition of oscillation is : $\theta=\theta_{R} \sin \left(\omega_{\theta} t\right), \theta_{R}=0.6$ degree, $\omega_{\theta}$ $=3.49 \mathrm{rad} / \mathrm{sec}$, where $\theta$ is the angular displacement and $\theta_{R}, \omega_{\theta}$ are amplitude of the angular displacement and angular frequency respectively. The pressure was measured by arranging the pressure sensors as shown in Fig.3.2. Pictures of the experiment were taken by $16 \mathrm{~mm}$ movie camera and the wave heights of the right and left

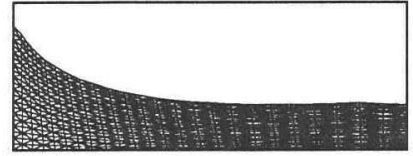

(a) $t=11.3 \mathrm{sec}$

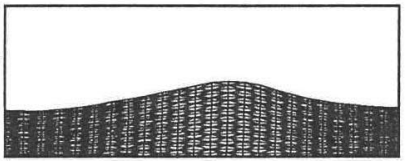

(b) $t=11.8 \mathrm{sec}$

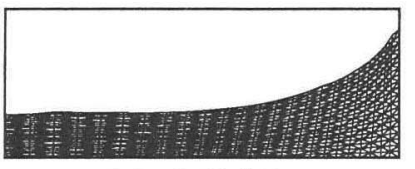

(c) $t=12.2 \mathrm{sec}$

Fig.3.4 Finite element configuration

walls were read from the film.

The finite element model is shown in Fig.3.4. The analytical region is divided into the mesh of 24 $\times 20$. The density is $1.0 \mathrm{Mg} / \mathrm{m}^{3}$ and the coefficient of kinematic viscosity is $\nu=1.0 \times 10^{-2} \mathrm{~cm}^{2} / \mathrm{sce}$. Time increment $\Delta t$ is $1.0 \times 10^{-3} \mathrm{sec}$. Boundary conditions are shown in Fig.3.2 and the acceleration effect of the rolling motion is taken into account as $f_{i}$ in equation (2.8).

Fig.3.3 shows the wave height of the right and left walls. The solid line shows the wave height from the $16 \mathrm{~mm}$ movie film of the experiment. The white circle shows the calculated result of the wave height. The calculated results agree well with the 


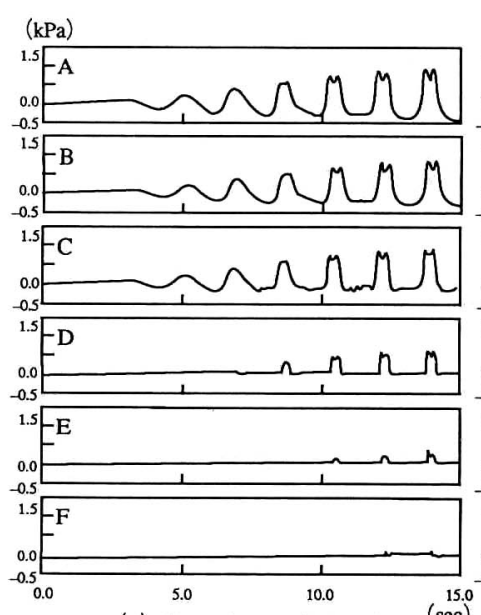

(a) Experimental Result

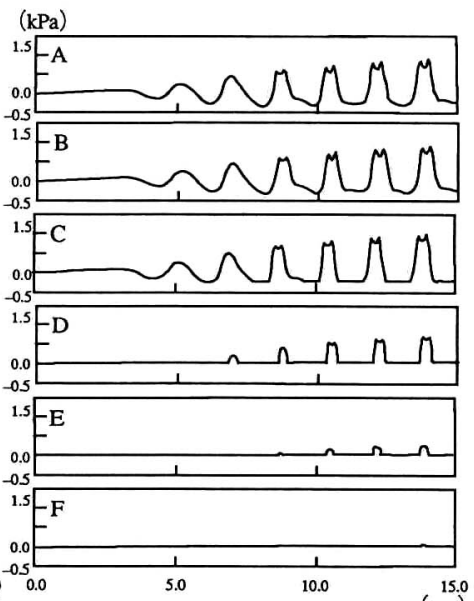

(b) Calculated Result

Fig.3.5 Measured and calculated pressure histories

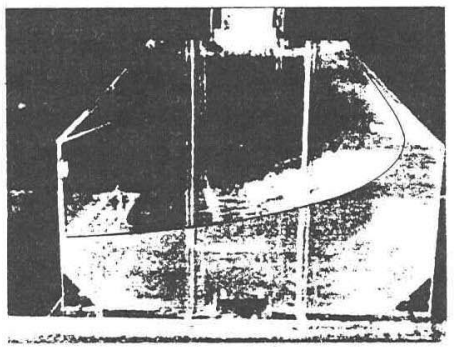

(a) Experimental result

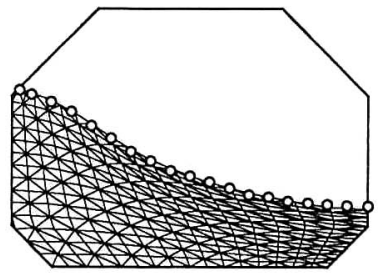

(b) Calculated result $(t=4.1 \mathrm{sec})$

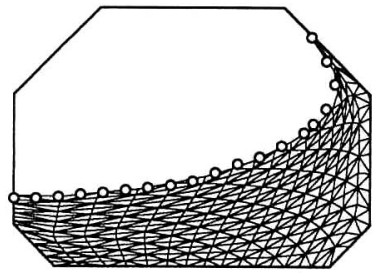

(c) Calculated result ( $t=4.8 \mathrm{sec})$

Fig.3.7 Experimental and calculated results at time $t=4.1 \mathrm{sec}$ and $t=4.8 \mathrm{sec}$

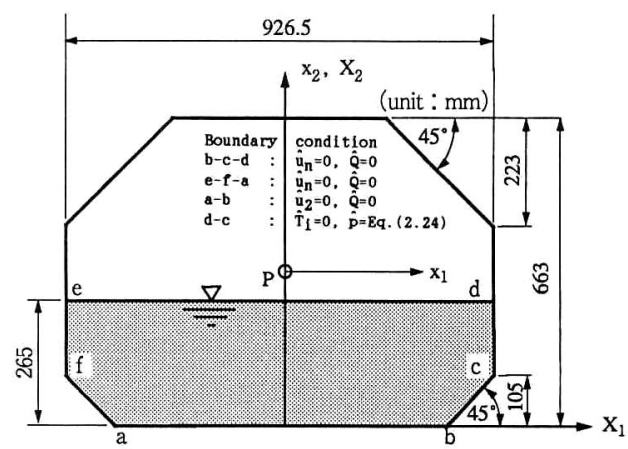

Fig.3.6 Model tank

experimental results. Calculated finite element configuration at time $t=11.3 \mathrm{sec}, 11.8 \mathrm{sec}$ and $12.2 \mathrm{sec}$ are shown in Fig.3.4 respectively. At time $t=12.2 \mathrm{sec}$, the ratio of sloshing wave height to water depth $\eta / h$ is 1.22. Fig.3.5 shows the measured and calculated pressure histories. The calculated pressure history agrees extremely well with the experimental one.

(3) COMPARISON WITH EXPERIMENT OF A TANK WITH ROOF AND CHAMFER

To show the adaptability of the present method, a comparison is made with the experimental results of a liquid motion in a tank which has roof and chamfer. The dimension of the tank is shown in Fig.3.6. Water is used and the depth is $265 \mathrm{~mm}$. The liquid region is divided into the mesh $16 \times 14$. The finite element model is shown in Fig.3.7. Density is $1.0 \mathrm{Mg} / \mathrm{m}^{3}$ and kinematic viscosity coefficient is $\nu=1.0 \times 10^{-2} \mathrm{~cm}^{2} / \mathrm{sec}$. A tank is oscillated by rotating, horizontal and perpendicular motions. The condition of the oscillation is : $X_{1}=$ $A_{X_{1}} \sin \left(\omega_{X_{1}} t\right)\left(A_{X_{1}}=61 \mathrm{~mm}, \omega_{X_{1}}=3.77 \mathrm{rad} / \mathrm{sec}\right), X_{2}$ $=A_{X_{2}} \sin \left(\omega_{X_{2}} t\right)\left(A_{X_{2}}=72 \mathrm{~mm}, \omega_{X_{2}}=3.77 \mathrm{rad} / \mathrm{sec}\right)$, $\theta=\theta_{R} \sin \left(\omega_{\theta} t\right)\left(\theta_{R}=3.0 \mathrm{deg}, \omega_{\theta}=2.90 \mathrm{rad} / \mathrm{sec}\right)$. The tank rotates around point $\mathrm{P}$, center of the tank. The time increment $\Delta t$ is $1.0 \times 10^{-3} \mathrm{sec}$. Boundary conditions are shown in Fig.3.6, and the acceleration effects of the motions are taken into account as the $f_{i}$ in equation (2.8). Experimental result at time $t=4.8 \mathrm{sec}$ and calculated results of the finite element configuration at time $t=4.1 \mathrm{sec}$ and $4.8 \mathrm{sec}$ are shown in Fig.3.7. In Fig.3.7 (b) and (c), white circles show the experiment's result. At time $t=4.8 \mathrm{sec}$, the configuration of the free surface is a double valued function of $x_{1}$. Even if the configuration of the free surface is a double 


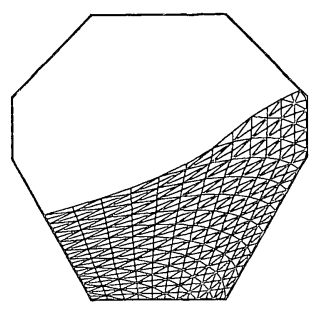

F.E. configuration

(a) At time $t=1.80 \mathrm{sec}$

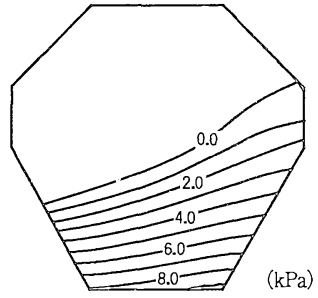

Pressure distribution

Fig.4.2 Analysis of liquid in a tank with roof and chamfer

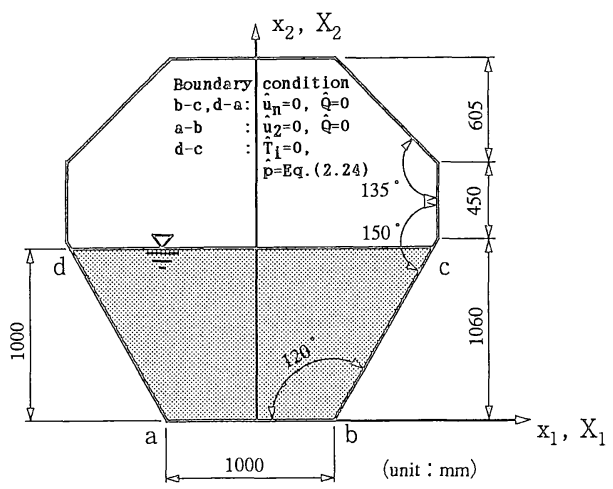

Fig.4.1 The dimensions of the tank

valued function, the calculated result completely agrees with the experimental result.

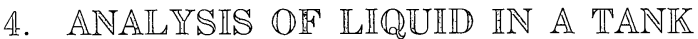

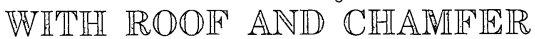

A liquid motion in a tank which has roof and chamfer is analyzed. The dimensions of the tank are shown in $\mathbb{F}$ ig.4.1. 1 . Liquid region is divided into the mesh of $12 \times 20$. Finite element model is shown in $\mathbb{F i g} .4 .2$. Density is $1.0 \mathrm{Mg} / \mathrm{m}^{3}$ and coefficient of kinematic viscosity is $\nu=1.0 \times 10 \mathrm{~cm}^{2} / \mathrm{sec}$. Acceleration $f_{2}$ is $g=-9.8 \mathrm{~m} / \mathrm{sec}^{2}$. At the initial stage, the tank is at rest and then moved in a sinusoidal way. Amplitude and period of displacement are $12.5 \mathrm{~cm}$ and $1.75 \mathrm{sec}$ respectively. The time increment $\Delta t$ in this calculation is $3.0 \times 10^{-3} \mathrm{sec}$. Boundary conditions are shown in $\mathbb{F i g . 4 . 1}$, and the acceleration effect of the motion is taken into account as $f_{1}$ in equation (2.8). Calculated results of the finite element configuration and pressure distribution are shown in Figs.4.2 (a) and (b). Sloshing wave can be shown to swell with time. At time $t=2.76 \mathrm{sec}$, because the wave covers almost all of the left upper chamfer, the configuration of the free surface is a double valued function of $x_{1}$. As the sloshing wave swells, the pressure distribution changes from the static pressure distribution. For example, at $t=2.76 \mathrm{sec}$ as shown in $\mathbb{F i g . 4 . 2}$ (b), the sloshing wave climbs up to the right wall

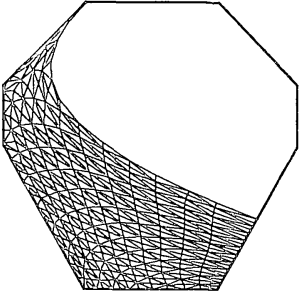

F.E. configuration (b) At timet $=2.76 \mathrm{sec}$

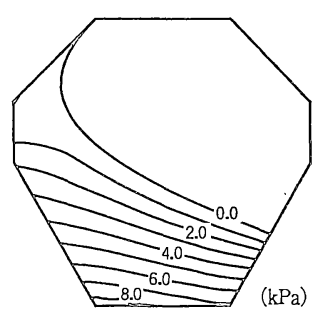

Pressure distribution and pressure contour lines become sparse in the left region and dense in the right region. In this calculation, it is possible to obtain the same result even if the time increment of 3 times as long as that in the Lagrangian finite element method $^{14)}$ is applied.

As shown in $\mathbb{F i g . 4 . 2}$, applying the ALE method the stable calculation of a large amplitude sloshing wave in a tank with roof and chamfer can be carried out.

\section{5. $\mathbb{C O N C L U S H O N S}$}

The outcome of the present paper can be summarized as follows.

(1) The new Arbitrary Lagrangian-Eulerian finite element method for the free surface flow and its application to a large amplitude sloshing analysis in a tank with roofs and/or chamfers are presented.

(2) The present method consists of three phases, i.e., Phase 1 : Purely Lagrangian calculation, Phase 2 : Remeshing procedure and Phase 3 : Rezoning procedure. In the calculation, a new fractional step method and a simple remeshing procedure are applied to overcome the main difficulty of the computation in a large amplitude sloshing problem.

(3) Comparison with the experimental results, the present method is shown to be sufficiently accurate to follow the free surface position and to obtain both velocity and pressure distributions.

(4) Numerical examples show that the stable calculation can be carried out and prove the validity of the present method to calculate the large amplitude sloshing waves even if the configuration of the free surface is a double-valued function.

(5) A refined mesh should be used in the practical application. The time increment which is 2 -3 times as long as that in the Lagrangian method can be used. Using the present method, the calculation can be carried out within the range of reasonable CPU time and memory size of a computer. From these results, it can be concluded that the present finite element method based on the Arbitrary Lagrangian-Eulerian method is useful 
and effective for the practical use of the analysis of the sloshing phenomena in a tank.

\section{ACKNOWLEDGMENT}

The authors wish to thank Dr. T.Nakayama, Messrs. K.Hatanaka and M.Hayashi, Chuo University for their helpful discussions and Messrs. T.Tanaka and S.Endo, NKK Corporation for their valuable support in performing the experiments shown in this paper.

\section{REFERENCES}

1) Harlow, F.H. and Welch, J.E. : Numerical Study of LargeAmplitude Free-surface Motions, Physics of Fluids, Vol.9, pp.842 851, 1966 .

2) Viecelli, J.A : A Computing Method for Incompressible Flows Bounded by Moving Walls, Journal of Computational Physics 8, pp.119 143, 1971.

3) Hirt, C.W, and Nichols, B.D. : Volume of Fluid (VOF) Method for the Dynamics of Free Boundaries, Journal of Computational Physics 39, pp.201 225, 1981.

4) Bridges, T.J. : A Numerical Simulation of Large Amplitude Sloshing, 3rd Int. Congress on Numerical Hydro dynamics, Paris, 1981.

5) Arai, M. : Experimental and Numerical Studies of Sloshing Pressure in Liquid Cargo Tank, Journal of The Society of Naval Architects of Japan, Vol.155, 1985.

6) Mikelis, N.E., Miller, J.K. and Taylor, K.V. : Sloshing in Partially Filled Liquid Tank and its Effect on Ship Motions : Numerical Simulations and Expermental Verification, The Naval Architect, RINA October, pp.267 281, 1984.

7) Hirt, C.W., Amsden, A.A. and Cook, J.L. : An Arbitrary Lagrangian-Eulerian Computing Method for all Flow Speeds, Journal of Computational Physics 14, pp.227 253, 1974.

8) Faltinsen, O.M. : A Numerical Nonlinear Method of Sloshing in Tanks with Two-Dimensional Flow, Journal of Ship Research Vol.22, No.3, pp.193 202, 1978.

9) Nakayama, T. and Washizu, K. : The Boundary Element Method Applied to the Analysis of Two-Dimensional Nonlinear Sloshing Problems, International Jouranl for Numerical Method in Engineering, Vol.17, pp.1631 1646, 1981

10) Nakayama, T. and Washizu, K. : Nonlinear Analysis of Liquid Motion in a Container Subjected to Forced Pitching Oscillation, International Journal for Numerical Methods in Engineering, Vol.15, No.8, pp.1207 1220, 1980.

11) Ohyama, T. : Nonlinear Sloshing Motion in a Circular Bashi, Journal of JSCE, No.417/ II -13, pp.255 264, 1990.

12) Ramaswamy, B., Kawahara, M. and Nakayama, T. : Lagrangian Finite Element Method for the Analysis of Two-Dimensional Sloshing Problems, International Journal for Numerical Methods in Fluids, Vol.6, pp.659 670, 1986.

13) Kawahara, M., Ramaswamy, B. and Anjyu, A. : Lagrangian Finite Element Method for Wave Motion Using Velocity Correction Method, Journal of JSCE, No.369/ II 5, pp.203 211, 1986.

14) Okamoto, T. and Kawahara, M. : Two-Dimensional Sloshing Analysis by Lagrangian Finite Element Method, International Journal for Numerical Methods in Fluids, Vol.11, pp.453 477, 1990.

15) Hughes, T.J.R., Liu, W.K. and Zimmerman, T.K. : Lagrangian-Eulerian Finite Element Formulation for Incompressible Viscous Flows, Computer Methods in Applied Mechanics and Engineering, 29, pp.329 349, 1981.

16) Donea, J., Giuliani, S. and Halleux, J.P. : An Arbitrary Lagrangian-Eulerian Finite Element Method for Transient Dynamic Fluid-Structure Interactions, Computer Method in Applied Mechanics and Engineering, 33, pp.689 723, 1982

17) Donea, J. : Arbitrary Lagrangian-Eulerian Finite Element Methods, Computational Methods for Transient Analysis pp.474 516, 1983.

18) Ramaswamy, B. and Kawahara, M. : Arbitrary Lagrangian-Eulerian Finite Element Method for Unsteady, Convective, Incompressible Viscous Free Surface Fluid Flow, International Journal for Numerical Methods in Fluids, Vol.7, pp.1053 1075, 1987.

19) Huerta, A. and Liu, W.K. : Viscous Flow with Large Free Surface Motion, Computer Method in Applied Mechanics and Engineering, 69, pp.277 324, 1988.

20) Huerta, A. and Liu, W.K. : Large-Amplitude Sloshing with Submerged Blocks, Transactions of the ASME, Journal of Pressure Vessel Technology, Vol.112, No.1, pp.104 108, 1990.

21) Nomura, T. and Iijima, M. : A Finite Element Procedure for Viscous Fluid-Structure Interaction Problems using the Arbitrary Lagrangian-Eulerian Finite Element Formulation, Journal of JSCE, No.416/I-13, pp.285 294, 1990.

(Received December 27, 1990)

\section{ALE 有限要素法による二次元スロッシング解析 \\ 岡本 隆・川原睦人 \\ 本論文では，屋根およびシャンファーを有するタンク内の大振幅スロッシング波解析 のための新しい ALE 有限要素法を提案した. 解析手法においては, 特に自由表面の境 界条件を満足するFractional Step 法を用いたこと, 幾つかの斜め壁で構成された境界 を取扱えることに特徴がある。提案手法は，実験結果との比較により，自由表面，流速， 圧力を求めるに十分な精度を有すること，また計算例により，自由表面が二価形状でも 解析可能なことを示した。}

